\title{
Universal entropy bound and discrete space-time
}

\author{
Yunqi Xu \\ School of Physics and State Key Laboratory of Nuclear Physics and Technology, \\ Peking University, Beijing 100871, China \\ Bo-Qiang Ma* \\ School of Physics and State Key Laboratory of Nuclear Physics and Technology, \\ Peking University, Beijing 100871, China \\ Center for High Energy Physics, Peking University, Beijing 100871, China and \\ Center for History and Philosophy of Science, Peking University, Beijing 100871, China
}

\begin{abstract}
Starting from the universal entropy bounds suggested by Bekenstein and Susskind and applying them to the black-body radiation situation, we get a cut-off of space $\Delta x \geq \chi l_{\mathrm{P}}$ with $\chi \geq 0.1$. We go further to get a cut-off of time $\Delta t \geq \chi l_{\mathrm{P}} / c$, thus, the discrete space-time structure is obtained. With the discrete space-time, we can explain the uncertainty principle. Based on the hypothesis of information theory and the entropy of black holes, we get the precise value of the parameter $\chi$ and demonstrate the reason why the entropy bounds hold.

PACS numbers: 04.20.Cv, 04.70.-s, 05.90.+m, 11.30.Cp
\end{abstract}

*Electronic address: mabq@pku.edu.cn 


\section{INTRODUCTION}

The generalized second law (GSL) and entropy bound came out with the development of black hole physics. In 1971, Hawking firstly derived the area theorem [1, 2], which states that the area of a black hole event horizon never decreases with time: $\mathrm{d} A \geq 0$. Inspired by this idea together with the second law in thermodynamics, Bekenstein [3 5] found that the area of a black hole takes the role of the entropy of the black hole and suggested that it is proportional to the entropy: $S_{\mathrm{BH}}=\alpha A$, where the proportional coefficient is derived by Hawking [6] to be $\alpha=1 / 4$. Moreover, Bekenstein also proposed that the second law of thermodynamics still holds for the total entropy including the black hole entropy and matter entropy outside it. This is the generalized second law: $\Delta S_{\text {total }} \geq 0$ and $S_{\text {total }}=S_{\text {matter }}+S_{\mathrm{BH}}$.

In classical general relativity, black holes can only absorb particles and never emit anything. However, in 1975, by means of semi-classical calculations of quantum field theory, Hawking [7] made a surprising discovery that black holes can create and emit particles like any other matter. It is just like a thermodynamic system with temperature $T=\kappa / 2 \pi$, in which $\kappa$ is the surface gravity of the event horizon. For a Schwarzschild black hole, the temperature is $1 / 8 \pi M$, which is inversely proportional to the mass of the black hole $M$. The area theorem is violated, however, the generalized second law still holds in this thermal emission situation.

The universal entropy bound is a direct inference of the generalized second law. In Section II, we present two different entropy bounds from Bekenstein and Susskind. They are equivalent to each other in many situations. Applying these two entropy bounds to the black-body radiation, we get two constraints for the temperature of the black-body. To be consistent with these two constraints, we draw a conclusion that the space has a minimum length scale proportional to the Planck length. A minimum time interval can also be obtained, thus, a discrete space-time structure is obtained. In Section III, we discuss the relation between the discrete space-time and the Heisenberg uncertainty principle. From information theory and a minimum volume introduced in Section IV, we derive a precise value of the parameter $\chi$. An explanation of the entropy bound is given in this section. Finally we discuss on the space-time discreteness and summarize the conclusions of our study. 


\section{ENTROPY BOUND AND DISCRETE SPACE-TIME}

By the analysis of the Geroch process, in which a system is dropped into a black hole from the vicinity of the event horizon, Bekenstein found that, if the generalized second law still holds, a universal entropy bound of the system is necessary. We call it the Bekenstein bound [8]:

$$
S_{\text {matter }} \leq 2 \pi E R
$$

in which $S$ is the entropy of the system, $E$ is the energy of it, and $R$ is the radius of smallest sphere that fits around the matter system.

Likewise, considering the Susskind process, in which the system shrinks and is converted to a black hole, Susskind [9, 10] argued that the generalized second law, if applied to the transformation, yields a spherical entropy bound:

$$
S_{\text {matter }} \leq \frac{A}{4}
$$

where $A$ is a suitably defined area enclosing the matter system. In the above two inequalities, only black holes can saturate the entropy bound, that is to say, at the same confined conditions, the black holes have the biggest entropy among all matter. So the above two inequalities are appropriate to all matter.

In the situation of a spherical system, both entropy bounds can be applied. Consider a spherical system filled with black-body radiation, in which the temperature is $T$ and the radius is $R$. From the thermodynamics of black-body radiation, we know that the entropy of the system is:

$$
S=\frac{4}{45} \pi^{2} T^{3} V=\frac{16}{135} \pi^{3} R^{3} T^{3} .
$$

Applying the entropy to the Susskind entropy bound $S \leq A / 4=\pi R^{2}$, we get a constraint of temperature $T$ :

$$
T \leq\left(\frac{135}{16 \pi^{2} R}\right)^{\frac{1}{3}}
$$

The larger the sphere is, the lower temperature the system has.

Similarly, the ratio of entropy-energy of black-body radiation satisfies:

$$
\frac{S}{E}=\frac{4}{3 T} \text {. }
$$

Substituting it to the Eq. (1), we get another constraint:

$$
T \geq \frac{2}{3 \pi R} .
$$


The smaller the sphere is, the higher temperature the system has, just like the previous one. By combining these two inequalities, the temperature must satisfy:

$$
\frac{2}{3 \pi R} \leq T \leq\left(\frac{135}{16 \pi^{2} R}\right)^{\frac{1}{3}} .
$$

To reconcile the two sides of the equation, the left side must be less than the right side, that is:

$$
\frac{2}{3 \pi R} \leq\left(\frac{135}{16 \pi^{2} R}\right)^{\frac{1}{3}} .
$$

We thus arrive at the following conclusion:

$$
R \geq\left(\frac{128}{3645 \pi}\right)^{\frac{1}{2}} l_{\mathrm{P}} \simeq 0.1 l_{\mathrm{P}},
$$

which means that space can not be infinitesimal. It must have a minimum value, which is proportional to the Planck length $l_{\mathrm{P}}=\sqrt{G \hbar / c^{3}}$. Therefore space is discrete rather than continuous. The minimum length scale is the cut-off of space, and it may eliminate the ultraviolet limit problem and the infinity in the quantum field theory. To be more universal, we set the proportional parameter to be $\chi$, that is, $\Delta x \geq \chi l_{\mathrm{P}}$ where $\chi \geq 0.1$.

As for time, we can also get a minimum time interval

$$
\Delta t=\frac{\Delta x}{v} \geq \frac{\Delta x}{c} \geq \frac{\chi l_{\mathrm{P}}}{c}=\chi \sqrt{\frac{G \hbar}{c^{5}}} .
$$

In the above derivation, we adopt the relation $v \leq c$, where $v$ is the velocity of an ordinary particle and $c$ is the velocity of light. If we adopt the natural unit system, i.e., we set $G=\hbar=c=k=1$, then $\Delta t \geq \Delta x \geq \chi$. The minimum volume of space is $\Delta V \geq \chi^{3}$. Therefore time has a minimum interval, which implies that the motion of a particle can not be divided to be infinitesimal but should be quantized. It is much like the transition from one quantum state to another one. As time is not infinitesimal, the time paradox put forward by Zeno can be solved easily.

\section{THE HEISENBERG UNCERTAINTY RELATION}

In quantum mechanics, we have the de Broglie relations:

$$
\begin{aligned}
& \lambda=h / p ; \\
& \nu=E / h .
\end{aligned}
$$


In the previous section we arrive at the conclusion that space-time must be discrete and get the relation of the smallest length of space:

$$
\Delta x \sim \chi l_{\mathrm{P}}
$$

which here we consider as the smallest uncertain length in quantum mechanics. The same discussion also applies to $\Delta t$. The uncertainty of the de Broglie wave length is almost the same size as the elementary length $\Delta \lambda \sim \chi l_{\mathrm{P}}$, and this means that the uncertain momentum of the particle is

$$
\Delta p=h / \Delta \lambda \sim h / \chi l_{\mathrm{P}}
$$

With these two relations, we immediately get the Heisenberg uncertainty relation

$$
\Delta x \Delta p \sim h .
$$

For another uncertainty relation about time and energy, we will divide it into two different situations. Firstly, we consider the non-relativistic particle. Using the relation implied by Badiali [11],

$$
\Delta p=m \Delta x / \Delta t,
$$

we have

$$
\Delta E=(\Delta p)^{2} / 2 m=\Delta p \Delta x / 2 \Delta t .
$$

Thus we get

$$
\Delta t \Delta E \sim \Delta x \Delta p / 2 \sim h / 2
$$

Otherwise, in the relativistic situation, there is a little difference:

$$
\begin{array}{r}
\Delta E=\Delta p c ; \\
\Delta t \Delta E \sim \Delta x \Delta p \sim h .
\end{array}
$$

To sum up, unlike the explanation in Ref. [11], where Badiali demonstrated the uncertainty principle from the discreteness of space-time and the set-up $(\Delta x)^{2} / \Delta t=\hbar / m$ issued from string theory, we get the two uncertainty relations $\Delta x \Delta p \sim h$ and $\Delta t \Delta E \sim h$ only from the discrete space-time derived in the above section. That means that quantum mechanics, especially the Heisenberg uncertainty principle is related closely with the discreteness of space-time structure. In the above discussion, we try to offer an understanding of the uncertainty principle from the viewpoint of discrete space-time, rather than a concrete proof. 


\section{INFORMATION AND BLACK HOLE ENTROPY}

The connection between information and entropy is well known. The entropy of a system measures the uncertainty or lack of information about the internal configuration of the system. Suppose all we know about a system are the probabilities $p_{n}$ corresponding to the $n$th state of the system. Then according to Shannon's entropy formula [12], the entropy associated with the system is:

$$
S=-\sum_{n} p_{n} \ln p_{n}
$$

The unit of information is bit, which is the information available when the answer to the yes-or-no question is known. So, according to the previous formula, the entropy of the information is maximized when the probability amplitude is $p_{\text {yes }}=p_{\text {no }}=1 / 2$. Thus, the entropy of one bit information is $\ln 2$.

It is widely believed that the information of an elementary system is one bit. Suppose the minimum volume of space can only accommodate the simplest binary system. That is to say that the maximum accommodation of information of the minimum volume is one bit. From a practical viewpoint, it is well-known that there is no pure plane without thickness, so the event horizon can be considered as a thin layer with $\Delta R=\chi l_{\mathrm{P}}$ as its thickness. Hence, the number of elementary systems is:

$$
N=\frac{4 \pi R^{2} \Delta R}{\left(\chi l_{\mathrm{P}}\right)^{3}}=\frac{4 \pi R^{2}}{\left(\chi l_{\mathrm{P}}\right)^{2}} .
$$

The entropy of one bit information is $\ln 2$. Multiplied with the unit of entropy $k_{\mathrm{B}}$, the entropy of the event horizon is:

$$
S=N k_{\mathrm{B}} \ln 2=\frac{k_{\mathrm{B}} A \ln 2}{\left(\chi l_{\mathrm{P}}\right)^{2}} .
$$

The entropy of the black hole to the outside observer is embodied in the event horizon and its value is $S=k_{\mathrm{B}} A / 4 l_{\mathrm{P}}^{2}$. Substituting the equation to Eq. (23), we obtain the numerical value of $\chi$ :

$$
\chi=2 \sqrt{\ln 2}
$$

which is consistent with the relation we get above: $\chi \geq 0.1$. Therefore, the accurate length of the minimum space is $2 \sqrt{\ln 2} l_{\mathrm{P}}=2.7 \times 10^{-35} \mathrm{~m}$, and the elementary area is $A_{0}=4 \ln 2 l_{\mathrm{P}}^{2}$, just the same as implied by 't Hooft in Refs. [13, 14]. In Ref. [15], it is suggested that a

length scale $l=l_{\mathrm{P}} / \eta$ might replace the gravity constant $G$ as a fundamental unit. Here we 
give a precise value of the parameter $\eta=1 / \chi=1 / 2 \sqrt{\ln 2}$. From that one can get a quantum gravity energy scale $E=\eta E_{\mathrm{P}}$ where $E_{\mathrm{P}} \equiv \sqrt{\hbar c^{5} / G}$ is the Planck energy. Such an energy scale is consistent with the gamma-ray burst observation [16] concerning the constraint on the Lorentz-violation scale [17, 18].

From section 2, we know that the highest temperature the black body can have is $T \leq$ $\left(135 / 16 \pi^{2} R\right)^{\frac{1}{3}} \leq 1.1 \times 10^{32} \mathrm{~K}$. Beyond this temperature, the discrete space-time structure might fuse and the physics there is unknown to us.

On the black hole event horizon, every elementary volume is an elementarily binary system, and this demonstrates the saturation of the entropy bound of the black hole. All these elementarily binary systems are fully quantized. It is natural to ask why other ordinary systems can not saturate the entropy bound? That may be because the elementary units on their boundaries are not fully quantized. This indicates that not all of them are binary systems, and it may explain why the entropy of an ordinary system is less than the entropy bound.

\section{COMMENTS AND SUMMARY}

In fact, the idea of space-time discreteness is not new, and there have been many arguments and debates from philosophical viewpoint in human history. Many discussions are well-known to us, such as Zeno's paradoxes, but most of them belong to the domain of metephysics. An origin of the discreteness of space-time from a physical viewpoint might be traced back to Planck, when he constructed a basic unit system in 1899 [19] before his idea of energy quanta, i.e., the birth of quantum theory in 1990. In Planck's unit system, there are five basic physical quantities, i.e., the light speed $c$, the gravitational constant $G$, the Boltzmann constant $k_{B}$, and $1 / 4 \pi \epsilon_{0}$ (where $\epsilon_{0}$ is the permittivity of free space or the electric constant), together with the new quantity $\hbar$ which is well-known as the Planck constant in quantum theory. There are a number of basic quantities in this unit system, such as the Planck length $l_{\mathrm{P}} \equiv \sqrt{G \hbar / c^{3}} \simeq 1.6 \times 10^{-35} \mathrm{~m}$, the Planck time $t_{\mathrm{P}} \equiv \sqrt{G \hbar / c^{5}} \simeq 5.4 \times 10^{-44} \mathrm{~s}$, the Planck energy $E_{\mathrm{P}} \equiv \sqrt{\hbar c^{5} / G} \simeq 2.0 \times 10^{9} \mathrm{~J}$, and the Planck temperature $T_{\mathrm{P}} \equiv \sqrt{\hbar c^{5} / G k_{B}^{2}} \simeq 1.4 \times 10^{32} \mathrm{~K}$.

As energy is quantized in quantum theory, the idea for an extension to the quantization of space and time has been discussed by many physicists afterward. There are have been many 
theories in which the concept of discreteness of space-time has been implemented, such as the non-commutative space-time [20]. Some developments of quantum gravity (QG) adopted a smallest length scale for the structure of space-time, or equivalently, an upper energy bound for particles in the quantum geometrical background [21 28]. The most natural candidate for the minimal length appears to be the Planck length $l_{\mathrm{P}}$, or accordingly, the Planck energy $E_{\mathrm{P}}$, for the maximal energy of particles. However, there have been suggestions [15] on the presence of a new fundamental length scale $l$, which, from dimensional analysis, is proportional to $l_{\mathrm{P}}$, i.e., $l=l_{\mathrm{P}} / \eta$, where $\eta$ corresponds to $1 / \chi=1 / 2 \sqrt{\ln 2}$ as pointed out in Section IV] of this paper.

Though a minimal length of space has been formulated in many theories, it is usually taken for granted as a hypothesis without physical explanation from a more concrete foundation. In this article, by applying the generalized second law and two universal entropy bounds suggested by Susskind and Bekenstein to the black-body radiation, we arrive at a minimum length scale of space. We also get the minimum time interval. Therefore, we reveal from physical arguments that space-time is discrete rather than continuous. Space and time can not be infinitesimal, but have elementary units.

We can explain the Heisenberg uncertainty relation using the discreteness of space-time. We also get the precise value of the elementary length. The highest temperature under which the space-time still holds is also obtained. The minimum length scale we got is a little different from the Planck length. It is a little larger than the Planck length many people thought before. Here we give the acquired minimum length scale more physically and concretely. The existence of a smallest length scale can manifest itself through the physical consequence of Lorentz invariance violation, which is a frontier under extensive investigations both theoretically and experimentally [29]. Thus the idea of discreteness of space-time can be tested through high precision measurements of Lorentz invariance violation effects.

From information theory, we can interpret the universal entropy bound and explain the inconsistency of entropies between black holes and ordinary systems. It is expected that fruitful results can be got from the new understanding of space-time discreteness.

Acknowledgments We thank Zhi Xiao and Lijing Shao for helpful discussions. This work is partially supported by National Natural Science Foundation of China (Grants No. 11021092, No. 10975003, No. 11035003). 
[1] S.W. Hawking, Phys. Rev. Lett. 26, 1344 (1971).

[2] S.W. Hawking, Comm. Math. Phys. 25, 152 (1972).

[3] J.D. Bekenstein, Nuovo Cim. Lett. 4, 737 (1972).

[4] J.D. Bekenstein, Phys. Rev. D 7, 2333 (1973).

[5] J.D. Bekenstein, Phys. Rev. D 9, 3292 (1974).

[6] S.W. Hawking, Nature 30, 248 (1974).

[7] S.W. Hawking, Commun. Math. Phys. 43, 199 (1975).

[8] J.D. Bekenstein, Phys. Rev. D 23, 287 (1981).

[9] L. Susskind, J. Math. Phys. 36, 6377 (1995) [arXiv: hep-th/9409089].

[10] R. Bousso, Rev. Mod. Phys. 74, 825 (2002) [arXiv: hep-th/0203101].

[11] J.P. Badiali, J. Phys. A 38, 2835 (2005) [arXiv: quant-ph/0409138].

[12] C.E. Shannon and W. Weaver, The Mathematical Theory of Communication. University of Illinois Press, Urbana (Ill.), 1949.

[13] G. 't Hooft, arXiv: gr-qc/9310026.

[14] G. 't Hooft, arXiv: hep-th/0003004.

[15] For a brief discussion and review on a minimal lenghth scale, see, e.g., L. Shao, B.-Q. Ma, arXiv: hep-th/1006.3031.

[16] A.A. Abdo et al., Nature 462, 331 (2009) arXiv:0908.1832.

[17] Z. Xiao, B.-Q. Ma, Phys. Rev. D 80, 116005 (2009) arXiv:0909.4927.

[18] L. Shao, Z. Xiao, B.-Q. Ma, Astropart. Phys. 33, 312 (2010). arXiv:0911.2276.

[19] M. Planck, Sitzungsberichte der Königlich Preußischen Akademie der Wissenschaften zu Berlin 5, 440 (1899).

[20] H.S. Snyder, Phys. Rev. 71 , 38 (1947); 72, 68 (1947).

[21] G. Amelino-Camelia, Phys. Lett. B 510, 255 (2001) arXiv:hep-th/0012238.

[22] G. Amelino-Camelia, Int. J. Mod. Phys. D 11, 35 (2002) arXiv:gr-qc/0012051.

[23] J. Kowalski-Glikman, Phys. Lett. A 286, 391 (2001) arXiv:hep-th/0102098.

[24] J. Kowalski-Glikman, Phys. Lett. A 299, 454 (2002) arXiv:hep-th/0111110.

[25] J. Magueijo and L. Smolin, Phys. Rev. Lett. 88, 190403 (2002) arXiv:hep-th/0112090.

[26] J. Magueijo and L. Smolin, Phys. Rev. D 67, 044017 (2003) arXiv:gr-qc/0207085].

[27] G. Amelino-Camelia, Int. J. Mod. Phys. D 11, 1643 (2002) arXiv:gr-qc/0210063.

[28] G. Amelino-Camelia, Symmetry 2, 230 (2010) arXiv:1003.3942 [gr-qc]].

[29] For a brief review on Lorentz violation effects through very high energy photons of astrophysical sources, see. e.g., L. Shao and B.-Q. Ma, Mod. Phys. Lett. A 25, 3251 (2010) arXiv:1007.2269 [hep-ph]]. 